\title{
Keynote Papers
}

\section{How will Electronic Health Records revolutionize healthcare?}

\author{
V. Wetekam*(volker.wetekam@siemens.com) \\ Siemens Medical Solutions - Global Solutions, Erlangen, Germany
}

\begin{abstract}
Throughout the centuries medical knowledge has been steadily extended. There have been many revolutional findings and theories. New methods and techniques in diagnosis and treatment have a strong impact on any humans personal health.

Since several years now another important development has taken place: sharing of medical knowledge by using the Internet. Yet this is only one step towards the bigger eHealth goal: providing information in time to individuals - consumers, patients, and professionals - when and where they need it, so they can use this information to make informed decisions about health and healthcare.

Siemens, having its over 150 years old roots in the healthcare technology as well as in communications business, has a strong vision for the future: eHealth will enable collaboration and a sector less cooperation between healthcare providers. This will result in higher quality and in the same time will reduce costs. The Electronic Healthcare Record (EHR) is an important cornerstone to achieve this goal.

In his keynote Dr. Volker Wetekam will outline this vision and will give project examples to show the pivotal role of Electronic Healthcare Records in cross-sectoral cooperations and in eHealth solutions for national healthcare programs.
\end{abstract}

Curriculum Vitae Volker Wetekam:

Volker Wetekam, born 1970 in Germany, studied Computer Science and Economics at the European Business School in Frankfurt, London and San Diego. He earned his Ph.D degree at the University of Leipzig, Germany, and completed an Executive Management Programme at the Duke University.

Before he was appointed CEO of Siemens Medical Solution Health Service in Germany, he had led the Consulting and Managed Service business within Siemens Medical Solutions. In September 2004 he was appointed President \& CEO of Global Solutions. He leads Siemens Medicals eHealth, Identity Management, PPP and Consulting business.

${ }^{*}$ These authors were submitted as lecturers of the presentation. 


\title{
Asklepios Future Hospital: OneIT - Largest IT Migration Project in Germany's Healthcare System
}

\author{
U. Pöttgen* (u.poettgen@asklepios.com) \\ Asklepios Clinics, Hamburg, Germany
}

ABSTRACT: The restructuring currently taking place in healthcare systems represents a host of new challenges for the healthcare sector - particularly hospitals and doctors. The study "Health Cast 2020" by PriceWaterhouseCoopers claims that many players in the healthcare sector will not survive the next 15 years unless significant changes are made.

By initiating the project Asklepios Future Hospital (AFH), the Asklepios Clinics have taken up these challenges in a broadly based long-term program. Together with key partners from industry, including founding partners Microsoft and Intel, from the healthcare system and organizations and associations. $\mathrm{AFH}$ is a development and testing ground for innovative solutions to enhance treatment quality, optimize processes and infrastructures and improve the cost-effectiveness of clinics.

A key condition for cost-effective operation of a future-proof clinic is a standardized IT infrastructure. Against this background, the lecture focuses on one of AFH's key projects: OneIT - the standardization of network and computer environments in Asklepios Kliniken Hamburg GmbH. The objective was to establish a uniform working environment for all staff while simultaneously increasing system performance, enhancing data security and system stability, and streamlining administration. The process was designed to simplify workflows, support inter-site work and bring sustainable cost reductions. Over 100 specialist departments, over 1,600 physicians and therapists and around 6,000 nursing and care staff at the six Asklepios clinics in Hamburg were affected by the changeover - the largest migration project in the German healthcare system to date. Completed in January 2007, the project proved to be a success. Its standardization processes slashed total costs per workstation, including "hidden costs" such as training courses and losses through downtime ("total cost of ownership") by 36.7 per cent.

The talk compares the situation at the clinics before and after migration, refers to the experiences gained and explains the benefits for cost-effective operation with the help of information from a study. In addition, the speaker describes how OneIT can be implemented in other clinics - for like all projects under the AFH heading, OneIT was designed specifically for use in other clinics too.

Successful implementation of OneIT is the most important foundation-stone for the implementation of further projects in AFH. In conclusion, the talk outlines further projects in Asklepios Future Hospital, such as the use of RFID technology, telemonitoring, or the physicians' portal for online communication between patients' private practitioners and the clinic.

\section{Curriculum Vitae Uwe Pöttgen:}

Being CIO at the Asklepios Group since 2005, Uwe Poettgen initialised the "Asklepios Future Hospital Programme". From 1996 until 2001, he was director of the IT/Controlling department at the Red Cross hospitals in Frankfurt.

Before he got promoted Director of Central Services IT at the Asklepios Group, Poettgen had worked as Director of IT at the Asklepios Clinics, where he was responsible for the region south-west Germany. 


\title{
Personal Health Records and Patient Empowerment
}

\author{
D. Albers*(dirk.albers@icw.de) \\ InterComponentWare AG (ICW AG), Walldorf/Baden, Germany
}

\begin{abstract}
The increasing empowerment of patients - becoming more and more educated consumers in the health care environment - will rely to a large extent on web based information and tools in the hands of citizens. This trend will continue to be one mayor driver in ehealth over the coming years.

Personal Health Records (PHR) will complete the triage of health records available for various purposes, e.g. in the hospital and office based area (EPRs), to further enable consumers to maneuver through the increasing, often overwhelming, health care offerings then based on qualified and reliable information provided by health care providers and other well acknowledged sources.

The keynote will elaborate on the underlying trends and mechanisms as well as detailing on the interconnectivity of records. It will finally, demonstrate already available solutions to consumers and present an outlook on future scenarios.
\end{abstract}

\section{Curriculum Vitae Dirk Albers:}

Before Dirk Albers took over management of InterComponentWare AG's Germany business, he was in charge of ICW's Corporate Business Development for a year. In his role as Managing Director Germany, he is responsible for ICW's Business Development, Marketing, Sales and Professional Service in Germany. Previously, he was a partner at IBM Business Consulting and held various marketing, sales and strategy positions at pharmaceutical companies in Germany (MSD) and the USA. Albers studied biomedical engineering and qualified as a graduate engineer at Aachen University of Applied Sciences, Germany, before earning a Master degree in International Business from the University of South Carolina, Columbia, USA, specializing in International Marketing.

\section{Online Health Communities}

\author{
L. Neal ${ }^{\mathrm{a}, \mathrm{b}}$ (lisa@acm.org) \\ a eLearn Magazine, New York, USA \\ ${ }^{\mathrm{b}}$ Tufts University School of Medicine, Boston, MA, USA
}

ABSTRACT: Online health communities provide a means for patients and their families to learn about an illness and seek support, or support others, in similar circumstances. The importance of online health communities is evidenced by their popularity, as well as the significant impact they have on the quality of life of their users. Online health communities raise difficult design challenges because of the wide variability of participant's medical expertise, health literacy, and technology literacy and the potential consequences when poor advice is taken or when professional treatment is not sought.

The criticality of health-related information necessitates careful consideration of how to design for usability and sociability. Furthermore, patients and their families may be under stress and the emotional burden, which can diminish health literacy, necessitates more careful design and evaluation. Disease and illness have no boundaries, and participants in online health communities can vary considerably in their knowledge about and need for education and support about a disease or condition.

Currently, there is no prevailing wisdom on best practices in online health community design and researchers have not yet conclusively demonstrated the health benefits of online health communities. 
Despite limited research on health benefits, there are many situations where online health communities appear to aid patients. These benefits include improved quality of life, better decision making, and patients who feel less alone and more empowered. The availability of online health communities is especially appreciated by individuals with impaired mobility, embarrassing conditions, or caretaker responsibilities that prohibit them from receiving adequate face-to-face medical and emotional support. At the same time, access and effective use can be restricted due to cultural, language, and other issues.

This talk will provide examples of online health communities along with anecdotal evidence and research findings. Furthermore this talk will explore how the nature of the disease or illness impacts the online health community design; how to improve health literacy; which of the many collaborative technologies that are available best support peer interaction; and how innovative Web 2.0 technologies can enhance people's ability to locate and contribute information to an online health community.

\section{Curriculum Vitae Lisa Neal:}

Lisa Neal is an Adjunct Assistant Clinical Professor of Public Health and Family Medicine at Tufts University School of Medicine, where she teaches a course on Online Health Communities. She is Editor-in-Chief of eLearn Magazine, published by ACM, and a consultant who designs and evaluates online communities and online courses for a variety of clients including Michael J Fox Foundation, Environment and Health Group, and the NIH. Lisa holds a Ph.D. in Computer Science from Harvard University. Her web site is at www.lisaneal.com. 